\title{
Correspondence
}

\section{Arterial oxygen saturation in cigarette smokers following general anaesthesia}

\section{To the Editor:}

A.R. Tait et al. ${ }^{\prime}$ in the May, 1990, issue of the Journal conclude, based on their Table III, that the "severity of hypoxaemia was significantly greater in the smoking group than in the non-smoking group."

Table III, as presented, is an example of rank ordered data, with smoking status as the independent variable and $\mathrm{SaO}_{2}$ as the dependent variable. I have recalculated the table to replace percentage by number of subjects.

TABLE III

\begin{tabular}{lllcc}
\hline & \multicolumn{4}{c}{$\mathrm{SaO}_{2}$} \\
\cline { 2 - 5 } & $\leq 84$ & $85-89$ & $90-94$ & $\geq 95$ \\
\hline Smokers & 3 & 3 & 7 & 6 \\
Non-smokers & 0 & 4 & 10 & 10 \\
\hline
\end{tabular}

The most appropriate statistical test for this data is the Mann-Whitney U test, ${ }^{2}$ which can be done manually and is included in most statistical packages. This test compares each of the smokers with each of the non-smokers, a total of $19 \times 24=456$ comparisons. If there is no difference half the comparisons $\left(U_{E}=228\right.$ ) should favour each group. The actual test statistic $U$, which is easily calculated as 273 , can be interpreted in two ways. First the probability that a smoker, chosen at random, will have a lower $\mathrm{SaO}_{2}$ than a non-smoker, also chosen at random, is $273 / 456$ or 0.6 . Not much greater than chance, which would be 0.5 . Secondly one can calculate the standard deviation and assess the statistical significance of $U$. The exact two-tailed probability is $0.25 ;^{3}$ the approximate $P$, calculated by hand, is 0.28 . Although the Chi-square and Fisher's exact tests are not appropriate for this data, they also lead to non-significant P-values. It is therefore difficult to understand how the authors have reached their conclusions.

With regard to Table $I 1$, they conclude that the "magnitude of the decrease in $\mathrm{SaO}_{2}$ was significantly greater in the smoking group." Although mean $\mathrm{SaO}_{2}$ values in the PACU look like they should be significantly different, the baseline values from the $O R$ are also different.

Most of us believe that cigarette smoking increases the risk of anaesthesia. Unfortunately this paper does not provide us with scientifically valid estimates of that risk. Although the mean $\mathrm{SaO}_{2}$ may be lower during transport in smokers, the probability of any randomly selected smoker being at greater risk than a non-smoker is too close to chance to serve as a criterion for therapeutic decisionmaking. Other factors must be considered in order to individualize treatment. If, based on this study, you advocate $\mathrm{O}_{2}$ for smokers, then rationally you must also advocate $\mathrm{O}_{2}$ for non-smokers.

\section{W.A. Tweed MD FRCPC \\ National University of Singapore}

\section{REFERENCES}

I Tait AR, Kyff JV, Crider $B$ el al. Changes in arterial oxygen saturation in cigarette smokers following general anaesthesia. Can J Anaesth 1990; 37: 423-8.

2 Moses LE, Emerson JD, Hasseini $H$. Analyzing data from ordered categories. N Engl J Med 1984; 311: 442-8.

3 Statgraphics, Statistical Graphics Corporation, 2115 East Jefferson St., Rockville, MD, USA 20852.

\section{Spinal narcotics}

To the Editor:

I am concerned about the current popularity of neuroaxial opioids and opiates in obstetrics as revealed in the editorial by Dr. Writer.' In my opinion, the danger of reactivation of previous serious CNS or ophthalmic herpes infection remains an unassessed, but potentially absolute, contraindication to the use of epidurally or intrathecally administered opioids or opiates. Given the rarity of serious extragenital infections none of the sample sizes of the studies done to date reassure me that reactivation of ophthalmic herpetic infection and its possible corneal destruction might not occur. Herpes keratitis is not unknown in the obstetric population and one can only speculate as to the risk in the immunocompromised obstetric patient.

Whether one is a "declining dowager" or a fecund female it appears to me to be a reasonable precaution to 